\title{
Influence Factors of Mobility-Related Activities of Daily Living in Chronic Dialysis Patients Needing Long-Term Care: Analysis of Long-Term Care Eligibility Assessment Survey Data
}

\author{
Utako Shimizu ${ }^{1 *}$, Hagiko Aoki ${ }^{1}$, Masaru Nakamura1 ${ }^{1}$, Haruo Yamanouchi' ${ }^{2}$, Kohei Akazawa ${ }^{3}$ \\ ${ }^{1}$ School of Sciences Faculty of Medicine, Niigata University, Niigata, Japan \\ ${ }^{2}$ Division of Legal Medicine, Niigata University Graduate School of Medical and Dental Science, Niigata, Japan \\ ${ }^{3}$ Department of Medical Informatics, Niigata University Medical and Dental Hospital, Niigata, Japan \\ Email: ${ }^{*}$ shirakaba@clg.niigata-u.ac.jp
}

Received 6 July 2014; revised 22 August 2014; accepted 7 September 2014

Copyright (C) 2014 by authors and Scientific Research Publishing Inc.

This work is licensed under the Creative Commons Attribution International License (CC BY). http://creativecommons.org/licenses/by/4.0/

(c) (i) Open Access

\section{Abstract}

Background: In the near future in Japan, chronic dialysis patients will likely face a situation where the aging and the lengthening of hemodialysis treatment periods will adversely affect the mobility-related activities of daily living (MR-ADL). The aim of this study was to investigate chronological change in MR-ADL among chronic dialysis patients in need of long-term care and the effect of admission to long-term care facilities on their MR-ADL. Methods: Data were analyzed from a longterm care eligibility assessment survey conducted in late March 2009 of 1000 individuals residing in Nigata City, Japan who were approved to receive long-term care. Then data from those individuals who had undergone the assessment survey $\geq 4$ times over a period of $\geq 4$ years were statistically evaluated. To reveal change in MR-ADL, this study performed a three-way analysis of variance with mean survey scores from assessment categories 2 - 5 containing questions related to MR-ADL as the independent variable and the number of eligibility assessment surveys (withinsubjects factor), facility admission, and dialysis therapy (between-subjects factors) as dependent variables. Results: We observed the effect of facility admission in individuals aged $>70$ years for categories 2 - 5, and observed the effect of the number of assessment surveys taken only in women $\geq 80$ years for categories 2 and 5 . Regarding the effect of dialysis therapy, female dialysis patients aged $\geq 80$ years and in need of long-term care scored significantly higher on survey items for transferring, grooming, toileting, eating, and instrumental activities of daily living, all of which are indicators of a moderate decline in ADL. Conclusions: Because dialysis is often initiated in women

\footnotetext{
${ }^{*}$ Corresponding author.
}

How to cite this paper: Shimizu, U., Aoki, H., Nakamura, M., Yamanouchi, H. and Akazawa, K. (2014) Influence Factors of Mobility-Related Activities of Daily Living in Chronic Dialysis Patients Needing Long-Term Care: Analysis of Long-Term Care Eligibility Assessment Survey Data. Health, 6, 2174-2183. http://dx.doi.org/10.4236/health.2014.616252 
aged around 80 years, ADL in this group of individuals can be improved by the initiation and proper provision of dialysis.

\title{
Keywords
}

\author{
Chronic Dialysis Patients, Activities of Daily Living, Long-Term Care Insurance, Long-Term Care \\ Insurance Facility
}

\section{Introduction}

In Japan, the mean age of chronic dialysis patients in late 2011 was 66.5 years, an increase of 4.9 years over 2001 figures. Over the same period, mean age at the time of dialysis onset had increased by 3.6 years, from 64.2 years in 2001 to 67.8 years in 2011 [1]. Thus, one of the characteristics of chronic dialysis in Japan is its aging patients. Another characteristic is a lengthening treatment period as patients age. As of 2011, the longest dialysis treatment period attained by a Japanese chronic dialysis patient was slightly over 43 years [1]. Long-term dialysis therapy is often accompanied by hemodialysis complications, especially abnormal bone metabolism and dialysis-related amyloidosis, causing bone and joint pain and joint dysfunction. In addition to frequent fatigue and blood pressure changes, bone and joint disorders can adversely affect the mobility-related activities of daily living (MR-ADL) of dialysis patients. Previous studies revealed that some ADL such as transferring, bathing, walking, and dressing are affected in elderly dialysis patients particularly [2], and dialysis onset is a factor that adversely affects the quality of ADL [3]. Therefore, the challenge associated with aging dialysis patients and lengthening dialysis treatment time is to develop measures against factors adversely affecting MR-ADL in dialysis patients.

Three major types of services are available for dialysis patients in need of long-term care: regular transportation to a medical facility provided by either a family member, the dialysis facility, or a special service facility under the long-term care insurance (LTCI) system; admission to a hospital for long-term care and rehabilitation under the health insurance system; and admission to a long-term care facility under the LTCI system. Dialysis patients needing long-term care and their families can choose among these services based on the patient's ADL and needs. However, the ADL of dialysis patients in need of long-term care and those factors affecting their ADL have not been fully elucidated. In Japan, since the establishment of the LTCI system in 2000, long-term care services are available upon approval to individuals aged >65 years. During the approval process, an 82-item eligibility assessment questionnaire is used to score the applicant's ADL, including MR-ADL.

The aim of this study was to use LTCI eligibility assessment scores to investigate chronological change in the MR-ADL of dialysis patients in need of long-term care and the effect of admission to a long-term care facility on their MR-ADL. Based on the results, we investigated support activities that are useful for maintaining MRADL in chronic dialysis patients.

\section{Methods}

\subsection{Long-Term Care Insurance (LTCI) System}

Japan implemented a long-term care insurance (LTCI) system in 2000 for every Japanese person aged $\geq 65$ strictly on the basis of physical and mental status [4]. Everyone aged $\geq 40$ years pays premiums, and everyone aged $\geq 65$ years is eligible for formal caregiving services [5]. Clients pay $10 \%$ of the eligible charges for services as a co-payment [6]. The older person (or caregiver) who wishes to receive long-term care applies to the public authority, and the initial assessment begins. The standard 2006 edition of the LTCI system assessment was used in this study, although as of 2013 the initial assessment is being carried out using the standard 2009 edition.

A trained local government official evaluates older people with long-term care needs during a home visit using a 70-item questionnaire on current physical and mental status and a 12-item questionnaire on the patient's use of medical procedures. This information obtained is then input into a computer program to estimate the applicant's standardized scores for seven dimensions of physical and mental status (scale 0 - 100). Table 1 shows these seven dimensions. 
Table 1. Eligibility assessment survey categories.

\begin{tabular}{cc}
\hline Category & Description \\
\hline Category 1 & Paralysis and limitation of joint movement \\
Category 2 & Physical movement and balance \\
Category 3 & Complex physical movement \\
Category 4 & Conditions requiring special assistance such as bedsores, lifting one arm, eating, toileting \\
Category 5 & Conditions requiring assistance with ADL/Instrumental ADL (IADL) \\
Category 6 & Communication and cognition \\
Category 7 & Behavioral and psychological problems \\
\hline
\end{tabular}

\subsection{Data}

Data from the long-term care eligibility assessment survey conducted in late March 2009 of 1000 individuals residing in Niigata City who were approved to receive long-term care were investigated in this study. Individuals who had undergone the eligibility assessment process $\geq 4$ times were divided into those who were dialysis patients (dialysis group) and those who were not (non-dialysis group). Table 2 shows the survey items. Figure 1 shows the results of statistical analysis.

\subsection{Statistical Analysis}

We evaluated MR-ADL using scores from categories 2 (Physical movement and balance), 3 (Complex physical movement), 4 (Conditions requiring special assistance as follows: bedsores, lifting one arm, eating, and toileting) and 5 (Conditions requiring assistance with ADL/instrumental ADL (IADL)) of the long-term care eligibility assessment survey. The maximum score for each category was 100 points, with higher scores signifying higher quality ADL. We grouped individuals by sex, age ( $\leq 69$ years, $70-79$ years, and $\geq 80$ years), admission to a long-term care facility, and dialysis therapy, and calculated the mean score of each group for the first to fourth assessment surveys. In this study, the oldest survey was defined as the first survey, and the latest survey was the fourth survey. We used the mean score for those who had taken more than one assessment survey within one year. To assess changes over time in ADL, three-way analysis of variance (ANOVA) was performed with mean score as the independent variable and the number of assessment surveys taken (within-subjects factor), facility admission, and dialysis therapy (between-subjects factors) as dependent variables according to the model below:

$$
Y_{i j k l}=\mu+\alpha_{i}+\beta_{j}+\alpha \beta_{i j}+\varepsilon_{i j l}^{1}+\gamma_{k}+\alpha \gamma_{i k}+\beta \gamma_{j k}+\alpha \beta \gamma_{i j k}+\varepsilon_{i j k l}^{2}
$$

$Y$ : Score of survey items

$M$ : Overall mean score

$\alpha_{i:}$ : Main effect of facility admission $i(i=1,2)$

$\beta_{j}$ : Main effect of dialysis therapy $j(j=1,2)$

$\gamma_{k}$ : Main effect of the number of eligibility assessment surveys $k(k=1,2,3,4)$

$\alpha \beta_{i j}$ : Interaction (facility admission $i$, dialysis therapy $j$ )

$\alpha \gamma_{i k}$ : Interaction (facility admission $i$, number of eligibility assessment surveys $k$ )

$\beta \gamma_{j k}$ : Interaction (dialysis therapy $j$, number of eligibility assessment surveys $k$ )

$\alpha \beta \gamma_{i j k}$ : Interaction (facility admission $i$, dialysis therapy $j$, number of eligibility assessment surveys $k$ )

$\varepsilon_{i j l}^{1}$ : Between-subject error term (assuming that both follow the normal distribution $N\left(0, \sigma_{1}^{2}\right)$ independently; $l=1,2, \cdots, N i j k)$

$\varepsilon_{i j k l}^{2}$ : Within-subject error term (assuming that both follow the normal distribution $N\left(0, \sigma_{2}^{2}\right)$ independently; $l=1,2, \cdots, N i j k)$

If no interaction effects were observed, two-way ANOVA was performed on the interaction between the number of surveys and dialysis therapy or between facility admission and dialysis therapy. Logarithmic conversion of scores was performed to approach their normal distributions. The statistical analysis software PASW18.0 was used for statistical analysis, with significance set at $p<0.05$. 
Table 2. Survey items used in this study.

\begin{tabular}{cc}
\hline No & Item \\
1 & Sex \\
2 & Age \\
3 & Scores from categories $2-5$ of the long-term care eligibility assessment survey \\
& (From the first LTCI assessment to March 31, 2009) \\
4 & Presence or absence of admission to a care facility \\
6 & Type of a care facility \\
7 & Fevel of assistance/care required \\
8 & Functional independence Physical disability \\
\hline
\end{tabular}

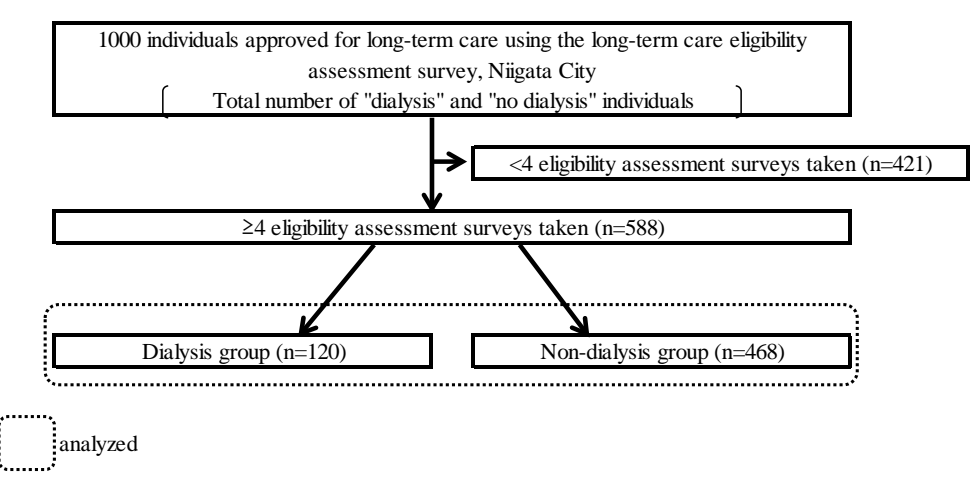

Figure 1. Data obtained and statistically analyzed. Data of $<4$ eligibility assessment surveys 1000 individuals, and divided into those who were dialysis group $(n=120)$ and those who were non-dialysis group $(n=468)$.

\subsection{Ethical Considerations}

The Niigata City Municipal Information Office approved the use of personal data provided that personal information was separated from the data. The Elderly Care Division under the Niigata City Health and Welfare Department stripped identifying information from the individual data and anonymized them with numbers before providing us with the data. This study was approved by the Ethics Committee of Niigata University School of Medicine.

\section{Results}

\subsection{Overview and Background of Subjects (Table 3)}

Of the 1000 individuals approved for long-term care as of March 31, 2009 in the study area, 1 person with incomplete data was excluded from this study. Of the remaining 999 individuals, 234 (113 men, 121 women; mean age, 77.9 years) were assigned to the dialysis group and the remaining 765 (356 men, 409 women; mean age, 84.4 years) were assigned to the non-dialysis group. Of these individuals, data from those who had undergone the eligibility assessment process for $\geq 4$ years were statistically evaluated. In the dialysis group $(n=120$; mean age, 77.9 years), individuals were further divided by age as follows: 24 individuals aged $\leq 69$ years (14 men, 10 women), 44 individuals aged 70 - 79 years ( 22 men, 22 women), and 52 individuals aged $\geq 80$ years (19 men, 33 women). At the time of the last survey, $35.1 \%$ of these individuals had been admitted to a medical facility by medical insurance and $45.6 \%$ to a long-term care facility by LTCI. In the non-dialysis group $(\mathrm{n}=468$; mean age, 84.7 years) who had undergone the eligibility assessment process for $\geq 4$ years consisted of 18 individuals aged $\leq 69$ years ( 10 men, 8 women), 114 individuals aged $70-79$ years (53 men, 61 women), and 336 
Table 3. Patient characteristics in the dialysis and non-dialysis groups.

\begin{tabular}{|c|c|c|c|c|c|}
\hline \multirow[b]{2}{*}{ Attribute } & \multirow[b]{2}{*}{ Classification } & \multicolumn{2}{|c|}{ Individuals approved for care } & \multicolumn{2}{|c|}{ Eligibility assessment survey for $\geq 4$ years } \\
\hline & & Dialysis $(\mathrm{n}=234)$ & $\begin{array}{l}\text { Non dialysis } \\
(\mathrm{n}=765)\end{array}$ & Dialysis $(\mathrm{n}=120)$ & $\begin{array}{l}\text { Non-dialysis } \\
(\mathrm{n}=468)\end{array}$ \\
\hline \multirow[t]{2}{*}{ Sex } & Men & $113(48.3)$ & 356 (46.5) & $55(45.8)$ & 186 (39.7) \\
\hline & Women & $121(51.7)$ & 409 (53.5) & $65(54.2)$ & $282(60.3)$ \\
\hline \multirow[t]{3}{*}{ Age (years) } & $\leq 69$ & $44(18.8)$ & $23(3.0)$ & $24(20.0)$ & $18(3.8)$ \\
\hline & $70-79$ & $91(38.9)$ & $215(28.1)$ & $44(36.7)$ & $114(24.4)$ \\
\hline & $\geq 80$ & 99 (42.3) & 527 (68.9) & $52(43.3)$ & 336 (71.8) \\
\hline \multirow[t]{2}{*}{ Facility admission } & Yes & - & - & $57(47.5)$ & $263(56.2)$ \\
\hline & No & - & - & $63(52.5)$ & $205(43.8)$ \\
\hline Type of facility & Health insurance facility & - & - & $20(35.1)$ & $40(15.2)$ \\
\hline Dialysis group $(\mathrm{n}=57)$ & $\begin{array}{l}\text { Long-term care insurance } \\
\text { facility }\end{array}$ & - & - & $26(45.6)$ & $179(68.1)$ \\
\hline $\begin{array}{l}\text { Non-dialysis group } \\
\qquad(\mathrm{n}=263)\end{array}$ & Other & - & - & $11(19.3)$ & 44 (16.7) \\
\hline \multirow[t]{7}{*}{$\begin{array}{l}\text { Level of assistance/care } \\
\text { required }\end{array}$} & Assistance level 1 & $2(0.9)$ & $43(5.6)$ & $0(0.0)$ & $19(4.1)$ \\
\hline & Assistance level 2 & 32 (13.7) & $64(8.4)$ & $13(10.8)$ & $33(7.1)$ \\
\hline & Care level 1 & $23(9.8)$ & 98 (12.8) & $15(12.5)$ & $41(8.8)$ \\
\hline & Care level 2 & $64(27.4)$ & 151 (19.7) & $26(21.7)$ & 99 (21.2) \\
\hline & Care level 3 & 55 (23.5) & $162(21.2)$ & $29(24.2)$ & $96(20.5)$ \\
\hline & Care level 4 & $40(17.1)$ & $138(18.0)$ & $25(20.8)$ & $95(20.3)$ \\
\hline & Care level 5 & $18(7.7)$ & $109(14.2)$ & $12(10.0)$ & $85(18.2)$ \\
\hline Functional independence & $\mathrm{J} 1$ & $5(2.1)$ & $9(1.2)$ & $2(1.7)$ & $3(0.6)$ \\
\hline \multirow[t]{7}{*}{ Physical disability } & $\mathrm{J} 2$ & $15(6.4)$ & $80(10.5)$ & $7(5.8)$ & $30(6.4)$ \\
\hline & A1 & $40(17.1)$ & $114(14.9)$ & 19 (15.7) & $60(12.8)$ \\
\hline & A2 & $68(29.1)$ & $220(28.8)$ & $29(24.8)$ & $125(26.7)$ \\
\hline & B1 & 44 (18.8) & $96(12.5)$ & 24 (19.8) & $64(13.7)$ \\
\hline & B2 & $31(13.2)$ & $130(17.0)$ & $22(18.2)$ & $100(21.4)$ \\
\hline & C1 & $13(5.6)$ & $27(3.5)$ & $7(5.8)$ & $19(4.1)$ \\
\hline & C2 & $18(7.7)$ & 89 (11.6) & $10(8.3)$ & $67(14.3)$ \\
\hline Functional independence & Independent & $57(24.4)$ & $74(9.7)$ & 27 (22.5) & $34(7.3)$ \\
\hline \multirow[t]{7}{*}{ Cognitive disability } & I & $66(28.2)$ & 137 (17.9) & $29(24.2)$ & 79 (16.9) \\
\hline & IIa & $29(12.4)$ & $74(9.7)$ & $17(14.2)$ & $39(8.3)$ \\
\hline & IIb & $43(18.4)$ & $177(23.1)$ & $24(20.0)$ & $108(23.1)$ \\
\hline & IIIa & $22(9.4)$ & $171(22.4)$ & 14 (11.7) & $111(23.7)$ \\
\hline & IIIb & $5(2.1)$ & $30(3.9)$ & $2(1.7)$ & $20(4.3)$ \\
\hline & IV & $10(4.3)$ & $86(11.2)$ & $5(4.2)$ & 63 (13.5) \\
\hline & $\mathrm{M}$ & $2(0.9)$ & $16(2.1)$ & $2(1.7)$ & $14(3.0)$ \\
\hline
\end{tabular}


individuals aged $\geq 80$ years (123 men, 213 women). Of these, $56.2 \%$ were admitted to a care facility: $15.2 \%$ to a medical facility by medical insurance and $68.1 \%$ to a long-term care facility by LTCI.

\subsection{Effect of facility admission revealed by ANOVA}

Scores from categories 2 - 5 of the long-term care eligibility assessment survey for patients aged $\geq 80$ years are shown Table 4. We observed no interaction of the three factors in any of categories 2 to 5 . In two-way ANOVA,

Table 4. Mean score of eligibility assessment survey (age $\geq 80$ years).

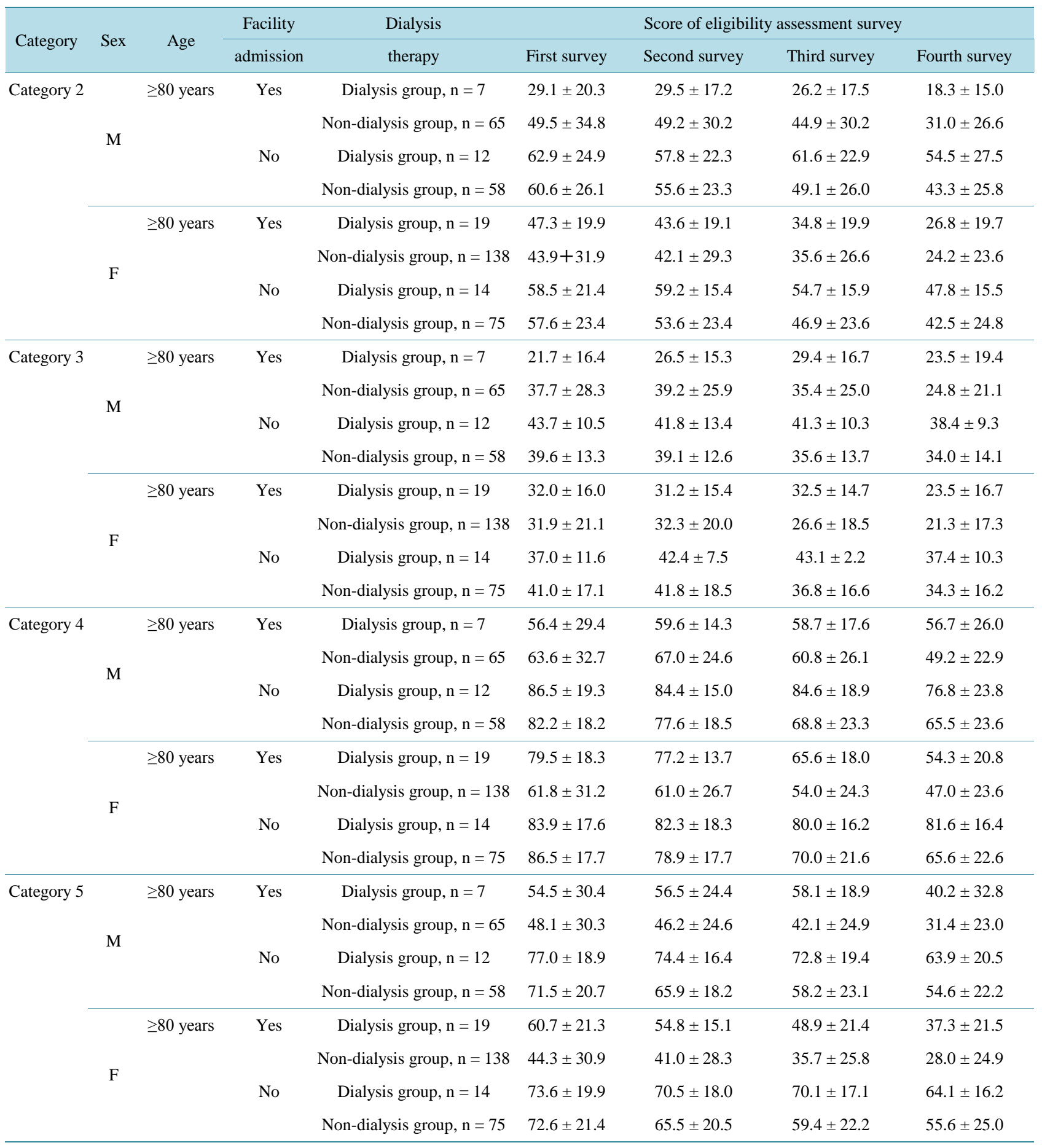


we observed no interaction between dialysis therapy and number of eligibility assessment surveys. However, we observed an interaction between facility admission and dialysis therapy only in women in their 70s for category $5(F, 4.809 ; d f 1 ; p<0.05)$. Regarding the simple main effect, individuals with no facility admission in the non-dialysis group had a significantly higher score $(F, 17.741 ; d f 1 ; p<0.001)$ (Figure 2, Table 5).

\subsection{Main Effect of the Number of Eligibility Assessment Surveys, Facility Admission, and Dialysis Therapy}

Table 6 shows the main effect of the number of eligibility assessment surveys, facility admission, and dialysis therapy. We observed the main effect of facility admission for individuals aged $\geq 70$ years for assessment categories 2 and 3 . We also observed the main effect for men in all age groups and women aged $\geq 70$ years for category 4 , and for men aged $\geq 70$ years and women aged $\geq 80$ years for category 5 . We observed the main effect of

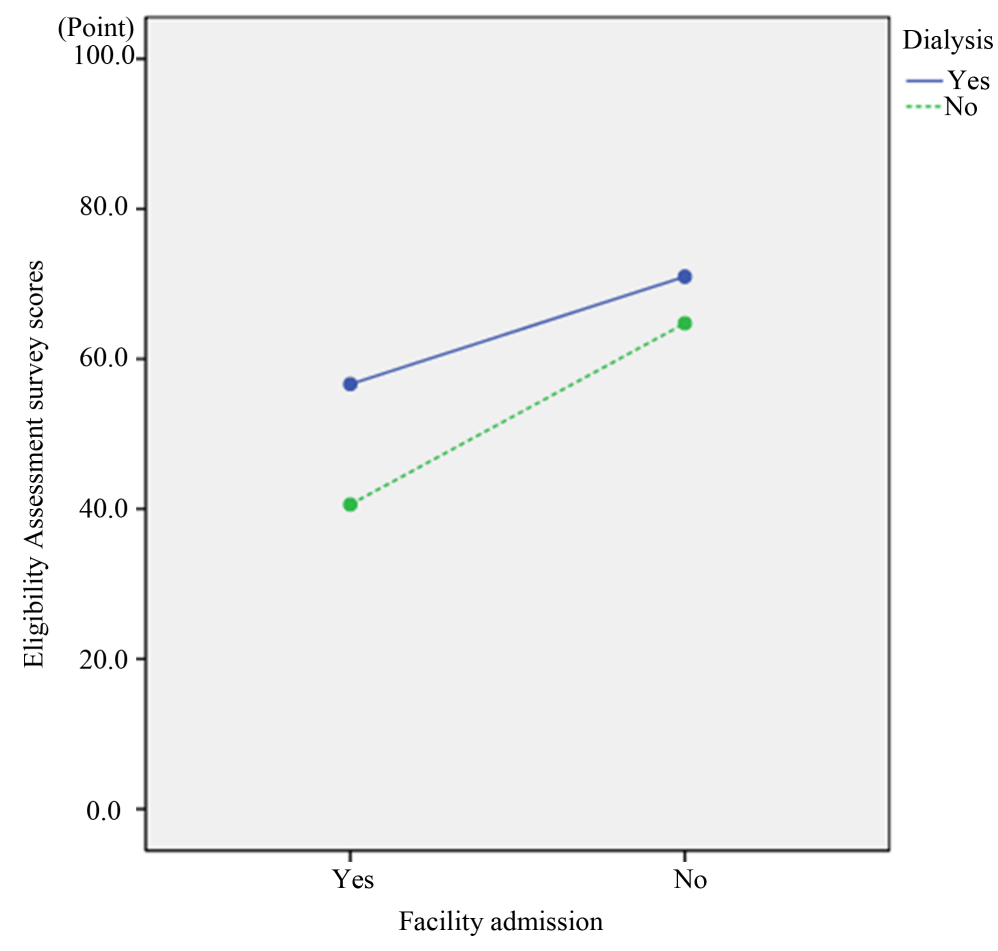

Figure 2. Interaction between facility admission and dialysis therapy (assessment category 5, women aged 70 - 79 years). An interaction was observed between facility admission and dialysis therapy. Then individuals with no facility admission in the non-dialysis group had a significantly higher score.

Table 5. Analysis of variance (category 5, women aged 70 - 79 years).

\begin{tabular}{|c|c|c|c|c|c|}
\hline Factors & Sum of squares & df & Mean square & F value & $p$ value \\
\hline Number of eligibility assessment surveys & 0.839 & 2.001 & 0.420 & 1.480 & 0.231 \\
\hline Facility admission & 8.841 & 1 & 8.841 & 4.171 & 0.044 \\
\hline Dialysis therapy & 8.453 & 1 & 8.453 & 4.809 & 0.049 \\
\hline Facility admission * dialysis therapy & 10.195 & 1 & 10.195 & 4.809 & 0.031 \\
\hline \multicolumn{6}{|l|}{ Simple main effect } \\
\hline Factors & Sum of squares & $\mathrm{df}$ & Mean square & F value & $p$ value \\
\hline Facility admission in the non-dialysis group & 9.401 & 1 & 9.401 & 17.741 & $<0.001$ \\
\hline
\end{tabular}


Table 6. Main effect of the number of assessment surveys, facility admission, and dialysis therapy.

\begin{tabular}{|c|c|c|c|c|c|c|c|}
\hline $\begin{array}{c}\text { Assessment } \\
\text { category }\end{array}$ & Sex, age & Factor & Sum of squares & $d f$ & Mean square & F value & $p$ value \\
\hline \multirow{6}{*}{ Category 2} & M, 70 - 79 years & Facility admission & 6.408 & 1 & 6.408 & 5.036 & 0.028 \\
\hline & $\mathrm{M}, \geq 80$ years & Facility admission & 8.844 & 1 & 8.844 & 15.31 & 0.000 \\
\hline & F, 70 - 79 years & Facility admission & 11.934 & 1 & 11.934 & 14.229 & 0.000 \\
\hline & $F, \geq 80$ years & Facility admission & 10.098 & 1 & 10.098 & 10.026 & 0.002 \\
\hline & & Dialysis therapy & 4.077 & 1 & 4.077 & 4.048 & 0.045 \\
\hline & & Number of surveys & 3.768 & 1.939 & 1.944 & 5.995 & 0.003 \\
\hline \multirow{4}{*}{ Category 3} & M, 70 - 79 years & Facility admission & 5.546 & 1 & 5.546 & 9.949 & 0.002 \\
\hline & $\mathrm{M}, \geq 80$ years & Facility admission & 4.584 & 1 & 4.584 & 12.641 & 0.001 \\
\hline & F, 70 - 79 years & Facility admission & 4.093 & 1 & 4.093 & 9.442 & 0.003 \\
\hline & $\mathrm{F}, \geq 80$ years & Facility admission & 6.568 & 1 & 6.568 & 14.967 & 0.000 \\
\hline \multirow{6}{*}{ Category 4} & $M, \leq 69$ years & Facility admission & 1.72 & 1 & 1.72 & 4.766 & 0.041 \\
\hline & M, 70 - 79 years & Facility admission & 3.287 & 1 & 3.287 & 7.586 & 0.007 \\
\hline & $\mathrm{M}, \geq 80$ years & Facility admission & 3.157 & 1 & 3.157 & 11.956 & 0.001 \\
\hline & F, 70 - 79 years & Facility admission & 1.875 & 1 & 1.875 & 5.599 & 0.020 \\
\hline & $\mathrm{F}, \geq 80$ years & Facility admission & 2.868 & 1 & 2.868 & 8.753 & 0.003 \\
\hline & & Dialysis therapy & 1.9 & 1 & 1.9 & 5.797 & 0.017 \\
\hline \multirow{5}{*}{ Category 5} & M, 70 - 79 years & Facility admission & 6.832 & 1 & 6.832 & 4.884 & 0.030 \\
\hline & $\mathrm{M}, \geq 80$ years & Facility admission & 8.283 & 1 & 8.283 & 9.369 & 0.003 \\
\hline & $\mathrm{F}, \geq 80$ years & Facility admission & 17.636 & 1 & 17.636 & 9.576 & 0.002 \\
\hline & & Dialysis therapy & 11.312 & 1 & 11.312 & 6.142 & 0.014 \\
\hline & & Number of surveys & 2.92 & 1.999 & 1.461 & 3.907 & 0.021 \\
\hline
\end{tabular}

dialysis therapy in women $\geq 80$ years for categories 2,4 , and 5 . As for the number of eligibility assessment surveys, we observed a main effect in women aged $\geq 80$ years for categories 2 and 5 .

\section{Discussion}

In this study, we observed no facility admission effect in individuals aged $<69$ years for assessment categories 2 , 3 , and 5 and in women aged $<69$ years for assessment category 4 . First-insured individuals aged $\geq 65$ years and in need of long-term care as well as individuals aged 40 - 64 years who have a specified disease (out of 16 specified diseases) are eligible for LTCI services. In this study, the limitation on the number of service users among individuals aged $\leq 64$ years might have contributed to the lack of main effects in groups of individuals aged $\leq 69$ years. Although we observed main and simple main effects of facility admission in individuals aged $\geq 70$ years for categories $2-5$, we observed the main effect of number of assessment surveys only in women aged $\geq 80$ years for categories 2 and 5, suggesting that, with or without dialysis, their ADL had already declined at the time of facility admission.

MR-ADL declines in various ways. In the elderly, a decline in ADL can start with ADL related to maintaining and changing posture, followed by leg function. As ADL declines further into the intermediate stage, IADL, dressing, transferring, grooming, toileting, eating, and communication are affected. In the advanced stage, swallowing is also affected, followed by memory and then cognitive function [7]. ADL in assessment categories 2 (maintaining and changing posture, mobility), 4 (bedsores, lifting one arm, eating, and toileting), and 5 (personal care, IADL) are generally affected in the intermediate stage. However, these ADL can be improved by dialysis 
treatment. As of 2008, the onset of dialysis in women peaks around age $75-79$ years (16.5\%), followed by 70 74 years $(15.3 \%)$ and $80-84$ years (13.6\%) [8]. In this study, we observed the main effect of dialysis among women aged $\geq 80$ years for categories 2,4 , and 5 . This might indicate that ADL in these female dialysis patients aged around 80 improved with dialysis therapy. These results therefore suggest that it is important for dialysis patients in need of long-term care to receive proper dialysis therapy and prevent symptoms that are associated with a moderate decline in ADL. By maintaining ADL in dialysis patients, it will be possible to reduce medical costs and the number of patients admitted for inpatient dialysis.

Living space affects the life of dialysis patients, including their activity level, personal care, and life rhythm, and therefore the care facility that they choose should meet their physical condition and medical care needs. Long-term care facilities in Japan are largely classified into three major types, special nursing homes for the elderly, geriatric health service facilities, and hospitals specified for long-term care and rehabilitation, with average room size per person being $13.2-10.65 \mathrm{~m}^{2}, 8 \mathrm{~m}^{2}$, and $6.4 \mathrm{~m}^{2}$, respectively [9]. The large size difference suggests that whereas rooms in the special nursing homes for the elderly are meant to be living spaces, rooms at the other two types of facilities serve as temporary accommodation for obtaining medical care and rehabilitation. However, the long-term care hospital system is scheduled to undergo simplification in 2017 when the LTCIbased hospital system terminates, thus leaving only the health insurance-based long-term care and rehabilitation system in place. A hospital specified for long-term care and rehabilitation that account for $35.1 \%$ of facility care in the dialysis group this study, will increase demand for dialysis patients. In the future, long-term care and rehabilitation hospitals need to provide services to manage the ADL of patients and to establish a system that allows patients to be transferred to other types of facilities or homes depending on their ADL.

Previous studies have reported on the benefits of exercise programs for maintaining MR-ADL in the elderly. Specifically, after engaging in a 4-month exercise program, elderly individuals aged $>75$ years had improved feelings of health with no change in physical strength [10], and a 12-month intervention improved the strength of the quadriceps muscles and maintained balancing and walking abilities in frail elderly women [11]. This latter training program was directed mainly at improving ADL such as maintaining and changing posture and mobility (in category 2) and standing up, maintaining a standing position on one leg, and bathing (in category 3 ). The effect of dialysis therapy was significantly higher in elderly women aged $>80$ years for category 2 . Women in other age groups and men in all age groups had similar ADL, with or without dialysis therapy, indicating the possibility of them obtaining effects similar to those of frail elderly women. Chronic dialysis patients often develop complications, and their physical condition can change quickly due to dialysis. Therefore, it is necessary to share patient information with their dialysis facility and provide and adjust training that is tailored to the physical condition of individual patients.

\section{Study Limitations}

This study analyzed data from dialysis patients in need of long-term care provided under the LTCI system. The result in this study basically could apply to only Japanese dialysis patients. Therefore, results might vary if the analysis were to include data from dialysis patients in need of long-term care but not using the LTCI system. Furthermore, this study also excluded data from dialysis patients in need of long-term care who were admitted to a hospital specified for long-term care and rehabilitation under the health insurance system. In the future, we plan to investigate MR-ADL by including dialysis patients who are in need of long-term care but do not take advantage of the LTCI system.

\section{Conclusion}

In this study, we used scores from categories 2 - 5 of the long-term care eligibility assessment survey, categories which evaluate the quality of MR-ADL, and calculated scores according to sex and age. We then performed three-way ANOVA with the number of eligibility assessment surveys as a within-subject factor and facility admission and dialysis therapy as a between-subjects factor. Although we observed the effect of facility admission among patients in their 70s and over 80 years of age for categories $2-5$, we observed the effect of the number of surveys taken only among female patients aged $\geq 80$ years for categories 2 and 5 . Regardless of dialysis therapy, patients who were in a facility must have had reduced ADL at the time of facility admission. In female dialysis patients aged $\geq 80$ years who were in need of long-term care, dialysis therapy significantly affected transferring, grooming, toileting, eating, and IADL, all of which are indicators of a moderate decline in ADL. Women aged 
around 80 years have a high rate of dialysis onset, and therefore initiation of dialysis and provision of appropriate dialysis therapy might improve their ADL. The results of this study suggest the importance of having dialysis patients who are in need of care maintain functional skills that are equivalent to mid-level ADL.

\section{Acknowledgements}

We thank the Elderly Care Division of Niigata City Health and Welfare Department for providing the long-term care eligibility assessment survey data used in this study.

\section{Competing Interests}

This study was supported by a Grant-in-Aid for Young Scientists (B) of the Ministry of Education, Culture, Sports, Science and Technology, Japan.

\section{References}

[1] The Japanese Society for Dialysis Therapy (2011) Current Status of Dialysis Therapy as of December 31, 2011: Illustrations. http://docs.jsdt.or.jp/overview/

[2] Farrokhi, F. and Jassal, S.V. (2013) Routine Use of an Abbreviated 4-Item Scale to Assess Dependence in Essential Activities of Daily Living amongst Elderly Hemodialysis Patients: A Validation Study. International Urology and Nephrology, 45, 259-264. http://dx.doi.org/10.1007/s11255-012-0360-4

[3] Kurella Tamura, M., Covinsky, K.E., Chertow, G.M., Yaffe, K., Landefeld, C.S., et al. (2009) Functional Status of Elderly Adults before and after Initiation of Dialysis. The New England Journal of Medicine, 361, 1539-1547. http://dx.doi.org/10.1056/NEJMoa0904655

[4] Tsutsui, T. and Muramatsu, N. (2005) Care-Needs Certification in the Long-Term Care Insurance System of Japan. Journal of the American Geriatrics Society, 53, 522-527. http://dx.doi.org/10.1111/j.1532-5415.2005.53175.x

[5] Tomata, Y. (2012) Green Tea Consumption and the Risk of Incident Functional Disability in Elderly Japanese: The Ohsaki Cohort 2006 Study. The American Journal of Clinical Nutrition, 95, 732-739. http://dx.doi.org/10.3945/ajcn.111.023200

[6] Tsutsui, T. and Muramatsu, N. (2007) Japan’s Universal Long-Term Care System Reform of 2005: Containing Costs and Realizing a Vision. Journal of the American Geriatrics Society, 55, 1458-1463. http://dx.doi.org/10.1111/j.1532-5415.2007.01281.x

[7] Japan Medical Association Research Institute (2004) Assessment of the Efficacy of Long Term Care Services. Report No. 65. Part 2, Current Situation and the Efficacy of Care Management by Multiple Insurers, Japan Medical Association Research Institute, Tokyo, 65-130.

[8] The Japanese Society for Dialysis Therapy (2008) Current Status of Dialysis Therapy as of December 31, 2008: Illustrations. http://docs.jsdt.or.jp/overview/index2009.html

[9] Health and Welfare Statistical Association (2005) Part 4: Health Insurance and Long Term Care Insurance. Chapter 2, The Long Term Care Insurance System: 2, Overview of the Long Term Care Insurance System; 3, Benefit Procedure and Description, Extra Edition. Journal of Health and Welfare Statistics, 52, 219-221. (in Japanese)

[10] Thompson, M., Lee, E., Hinomizu, A. and Nakamura, Y. (2005) Development of an Exercise Program for the Improvement of QOL (and for Care Prevention) in the Elderly over 75 Years of Age. Sport Science Research, 2, 113-121. (in Japanese)

[11] Ikezoe, T., Tsutou, A., Asakawa, Y. and Tsuboyama, T. (2005) Low Intensity Training for Frail Elderly Women: Long-Term Effects on Motor Function and Mobility. Journal of Physical Therapy Science, 17, 43-49. http://dx.doi.org/10.1589/jpts.17.43 
Scientific Research Publishing (SCIRP) is one of the largest Open Access journal publishers. It is currently publishing more than 200 open access, online, peer-reviewed journals covering a wide range of academic disciplines. SCIRP serves the worldwide academic communities and contributes to the progress and application of science with its publication.

Other selected journals from SCIRP are listed as below. Submit your manuscript to us via either submit@scirp.org or Online Submission Portal.
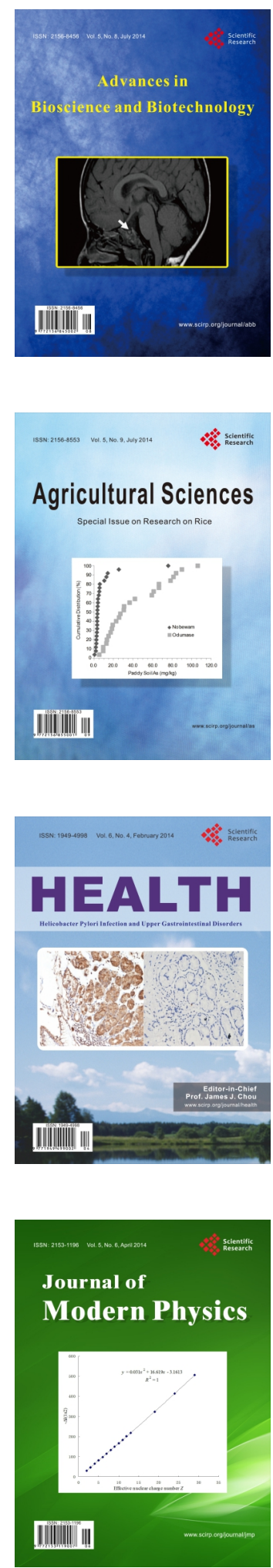
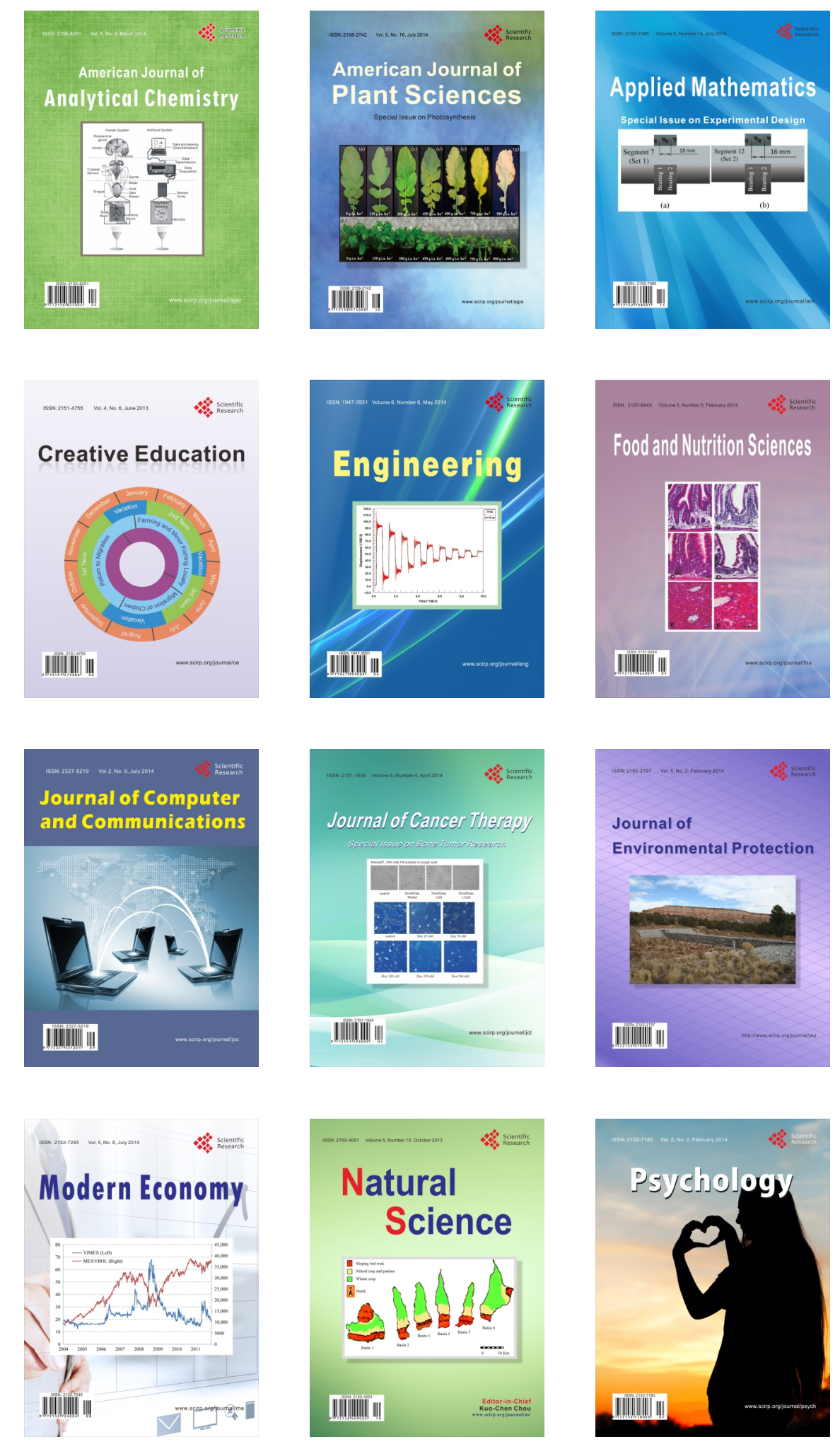